\title{
Deviation of Stellar Orbits From Test Particle Trajectories Around Sgr A* Due to Tides and Winds
}

\section{Citation}

Psaltis, Dimitrios, Gongjie Li, and Abraham Loeb. 2013. "DEVIATION OF STELLAR ORBITS FROM TEST PARTICLE TRAJECTORIES AROUND SGr A* DUE TO TIDES AND WINDS.” The Astrophysical Journal 777 (1): 57. https://doi.org/10.1088/0004-637x/777/1/57.

\section{Permanent link}

http://nrs.harvard.edu/urn-3:HUL.InstRepos:41393178

\section{Terms of Use}

This article was downloaded from Harvard University's DASH repository, and is made available under the terms and conditions applicable to Other Posted Material, as set forth at http:// nrs.harvard.edu/urn-3:HUL.InstRepos:dash.current.terms-of-use\#LAA

\section{Share Your Story}

The Harvard community has made this article openly available.

Please share how this access benefits you. Submit a story.

Accessibility 


\title{
DEVIATION OF STELLAR ORBITS FROM TEST PARTICLE TRAJECTORIES AROUND SGr A* DUE TO TIDES AND WINDS
}

\author{
Dimitrios Psaltis ${ }^{1,2}$, GongJie Li $^{2}$, And Abraham Loeb ${ }^{2}$ \\ ${ }^{1}$ Astronomy Department, University of Arizona, 933 North Cherry Avenue, Tucson, AZ 85721, USA; dpsaltis@email.arizona.edu \\ 2 Institute for Theory \& Computation, Harvard-Smithsonian CfA, 60 Garden Street, Cambridge, MA, USA; gli@cfa.harvard.edu, aloeb@cfa.harvard.edu \\ Received 2012 December 13; accepted 2013 April 4; published 2013 October 17
}

\begin{abstract}
Monitoring the orbits of stars around Sgr A* offers the possibility of detecting the precession of their orbital planes due to frame dragging, of measuring the spin and quadrupole moment of the black hole, and of testing the no-hair theorem. Here we investigate whether the deviations of stellar orbits from test-particle trajectories due to wind mass loss and tidal dissipation of the orbital energy compromise such measurements. We find that the effects of stellar winds are, in general, negligible. On the other hand, for the most eccentric orbits $(e>0.96)$ for which an optical interferometer, such as GRAVITY, will detect orbital plane precession due to frame dragging, the tidal dissipation of orbital energy occurs at timescales comparable to the timescale of precession due to the quadrupole moment of the black hole. As a result, this non-conservative effect is a potential source of systematic uncertainty in testing the no-hair theorem with stellar orbits.
\end{abstract}

Key words: black hole physics - Galaxy: center

Online-only material: color figures

\section{INTRODUCTION}

Stars in orbit around the black hole in the center of the Milky Way, hereafter Sgr A*, have been tracked for more than a decade, providing a measure of the black hole mass (Genzel et al. 2010; Ghez et al. 2012). The constraints have been steadily improving with the first measurement of a fully closed orbit for the star S2 (see, e.g., Ghez et al. 2008; Gillessen et al. 2009) as well as the discovery of additional stars (S0-16, S0-102, and S0-104) in orbits that probe the black-hole spacetime within a few thousand gravitational radii (Meyer et al. 2012).

Precise astrometric observations of stars in close orbits around Sgr A* may lead to the detection of orbital precession due to general relativistic frame dragging, measuring the spin of the black hole, and testing the no-hair theorem (Will 2008). Such measurements will be complementary to those that will be achieved with the Event Horizon Telescope (Fish \& Doeleman 2010; Johannsen \& Psaltis 2010) as well as to timing observations of pulsars in orbit around the black hole (Pfahl \& Loeb 2004; Liu et al. 2012).

Future instruments, such as GRAVITY, an adaptive-opticsassisted interferometer on the Very Large Telescope (Eisenhauer et al. 2011), will track stellar orbits with a single pointing astrometric accuracy of $\simeq 10-200 \mu \operatorname{arcsec}$, for stars as faint as $m_{\mathrm{K}}=16.3-18.8$ in a crowded field (Stone et al. 2012). At this resolution, the biggest challenge in measuring the fundamental properties of Sgr $\mathrm{A}^{*}$ with stellar orbits will be ensuring that a particular measurement is not affected adversely by astrophysical complications.

A number of studies have explored the effects of nongravitational forces exerted on the orbiting stars by other objects in the same environment. Merritt et al. (2010) and Sadeghian \& Will (2011) investigated the perturbative effects of the stellar cluster on the orbits of individual stars and found that they are negligible compared to the general relativistic effects inside $\sim 1 \mathrm{mpc} \simeq 5 \times 10^{3}$ gravitational radii. Psaltis (2012) studied the interaction of the orbiting stars with the ambient gas and showed that hydrodynamic drag and star-wake interactions are negligible inside $\sim 10^{5}$ gravitational radii.
In this paper, we study the deviations of the stellar orbits from test-particle trajectories that are introduced by the fact that stars are not point particles but (1) may lose mass in strong winds and (2) may be tidally deformed. We calculate the range of orbital parameters for which orbital perturbations due to the stellar winds and tides do not preclude the measurement of the black-hole spin and quadrupole moment and, therefore, testing of the no-hair theorem.

\section{CHARACTERISTIC TIMESCALES}

We start by comparing the characteristic timescales for orbital precession due to general relativistic effects to those of orbital perturbations due to stellar winds and to tidal forces. Hereafter, we set the mass of the black hole to $4 \times 10^{6} M_{\odot}$ and its distance to $8.4 \mathrm{kpc}$. We also denote by $M_{\mathrm{BH}}$ the mass of the black hole, by $M_{\mathrm{S}}$ the mass of the star, and by $a$ and $e$ the semimajor axis and eccentricity, respectively, of the stellar orbit. With these definitions, the Newtonian period of a stellar orbit is

$$
\begin{aligned}
P & =2 \pi\left(\frac{a^{3}}{G M_{\mathrm{BH}}}\right)^{1 / 2} \\
& =123.8\left(\frac{M_{\mathrm{BH}}}{4 \times 10^{6} M_{\odot}}\right)\left(\frac{a c^{2}}{G M_{\mathrm{BH}}}\right)^{3 / 2} \mathrm{~s} .
\end{aligned}
$$

\subsection{Dynamical Timescales}

General relativistic corrections to Newtonian gravity affect the orbits of stars around Sgr A* in at least three ways.

First, eccentric orbits precess on the orbital plane (periapsis precession). The characteristic timescale for this precession is (Merritt et al. 2010)

$$
\begin{aligned}
t_{\mathrm{S}} & =\frac{P}{6} \frac{c^{2} a}{G M_{\mathrm{BH}}}\left(1-e^{2}\right) \\
& =20.63\left(\frac{M_{\mathrm{BH}}}{4 \times 10^{6} M_{\odot}}\right)\left(\frac{a c^{2}}{G M_{\mathrm{BH}}}\right)^{5 / 2}\left(1-e^{2}\right) \mathrm{s} .
\end{aligned}
$$




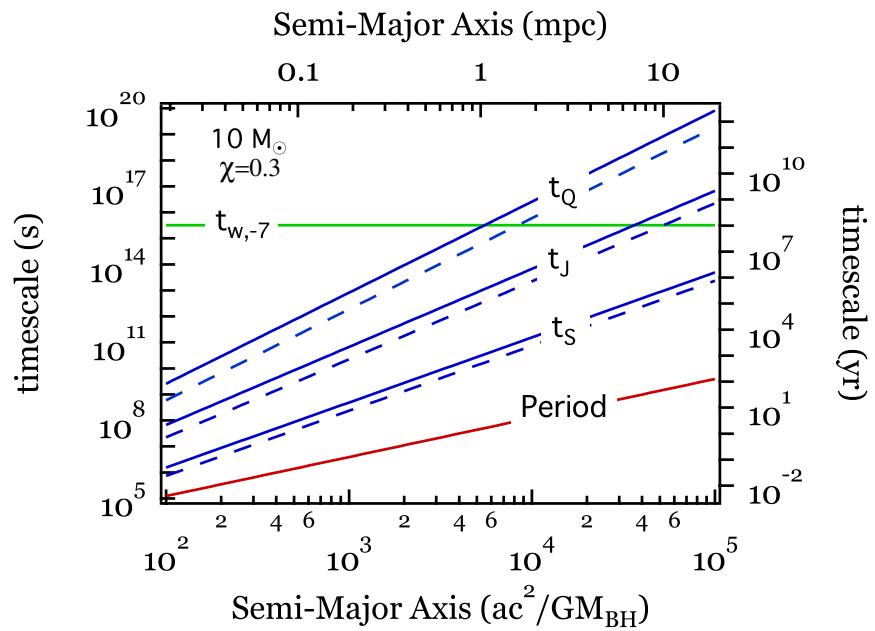

Figure 1. Different timescales that are relevant to the evolution of orbits of stars in the vicinity of $\operatorname{Sgr} \mathrm{A}^{*}$, as a function of their semimajor axes. The red line shows the periods of the orbits. The blue lines show the timescales for the precession of the periapsis $\left(t_{\mathrm{S}}\right)$, for the precession of the orbital plane due to frame dragging $\left(t_{\mathrm{J}}\right)$, and for the precession of the orbital frame due to the quadrupole moment of the spacetime $\left(t_{\mathrm{Q}}\right)$; the black hole spin is taken to be $\chi=0.3$ and solid and dashed lines correspond to eccentricities of 0.5 and 0.8 , respectively. The green line $\left(t_{\mathrm{w},-7}\right)$ shows the characteristic timescale for orbital evolution of a $10 M_{\odot}$ star due to the presence of a stellar wind at a mass-loss rate of $10^{-7} M_{\odot} \mathrm{yr}^{-1}$.

(A color version of this figure is available in the online journal.)

Second, orbits with angular momenta that are not parallel to the spin angular momentum of the black hole precess because of frame dragging. The characteristic timescale for this precession is (Merritt et al. 2010)

$$
\begin{aligned}
t_{\mathrm{J}} & =\frac{P}{4 \chi}\left[\frac{c^{2} a\left(1-e^{2}\right)}{G M_{\mathrm{BH}}}\right]^{3 / 2} \\
& =30.95 \chi^{-1}\left(\frac{M_{\mathrm{BH}}}{4 \times 10^{6} M_{\odot}}\right)\left(\frac{a c^{2}}{G M_{\mathrm{BH}}}\right)^{3}\left(1-e^{2}\right)^{3 / 2} \mathrm{~s}
\end{aligned}
$$

where $\chi$ is the spin of the black hole.

Finally, tilted orbits also precess because of the quadrupole moment of the spacetime. The characteristic timescale for this precession is (Merritt et al. 2010)

$$
\begin{aligned}
t_{\mathrm{Q}} & =\frac{P}{3|q|}\left[\frac{c^{2} a\left(1-e^{2}\right)}{G M_{\mathrm{BH}}}\right]^{2} \\
& =41.26|q|^{-1}\left(\frac{M_{\mathrm{BH}}}{4 \times 10^{6} M_{\odot}}\right)\left(\frac{a c^{2}}{G M_{\mathrm{BH}}}\right)^{7 / 2}\left(1-e^{2}\right)^{2} \mathrm{~s},
\end{aligned}
$$

where $q$ is the quadrupole moment of the black-hole spacetime. If the spacetime of the black hole satisfies the no-hair theorem, then $q=-\chi^{2}$.

The three timescales for a spinning Kerr black hole $(\chi=0.3$, $\left.q=-\chi^{2}\right)$ and for orbits with two different eccentricities are shown in Figure 1 as a function of the orbital semimajor axis.

\subsection{Wind Mass Loss}

The energy and angular momentum of a star in orbit around the more massive black hole are

$$
E=-\frac{G M_{\mathrm{BH}} M_{\mathrm{S}}}{2 a}
$$

and

$$
J=M_{\mathrm{S}}\left[G M_{\mathrm{BH}}\left(1-e^{2}\right) a\right]^{1 / 2},
$$

respectively. We will assume that the star is losing mass in a wind at a rate $\dot{M}_{\mathrm{w}}<0$ and that the wind is carrying a fraction $\xi$ of the orbital energy and a fraction $\lambda$ of the orbital angular momentum, i.e.

$$
\dot{E}=-\dot{E}_{\mathrm{w}}=\xi\left|\dot{M}_{\mathrm{w}}\right|\left(\frac{G M_{\mathrm{BH}}}{2 a}\right)
$$

and

$$
\dot{J}=-\dot{J}_{\mathrm{w}}=-\lambda\left|\dot{M}_{\mathrm{w}}\right|\left[G M_{\mathrm{BH}} a\left(1-e^{2}\right)\right]^{1 / 2} .
$$

With these definitions, a wind that is launched isotropically on the surface of the star will correspond to $\xi=\lambda=1$.

The removal of energy and angular momentum from the orbit causes, in principle, both its semimajor axis and its eccentricity to evolve, since

$$
\frac{\dot{E}}{E}=\frac{\dot{M}_{\mathrm{w}}}{M_{\mathrm{S}}}-\frac{\dot{a}}{a}
$$

and

$$
\frac{\dot{J}}{J}=\frac{\dot{M}_{\mathrm{w}}}{M_{\mathrm{S}}}+\frac{\dot{a}}{2 a}-\frac{e \dot{e}}{1-e^{2}} .
$$

Combining Equations (5)-(10) we can calculate the rate of change of the orbital separation:

$$
\frac{\dot{a}}{a}=-(1-\xi)\left|\frac{\dot{M}_{\mathrm{w}}}{M_{\mathrm{S}}}\right|
$$

and the rate of change of the orbital eccentricity:

$$
\frac{2 e \dot{e}}{1-e^{2}}=(\xi+2 \lambda-3)\left|\frac{\dot{M}_{\mathrm{w}}}{M_{\mathrm{S}}}\right| \text {. }
$$

In other words, we can define a characteristic timescale for orbital evolution due to the presence of the wind as

$$
\tau_{\mathrm{w}} \equiv\left|\frac{M_{\mathrm{S}}}{\dot{M}_{\mathrm{w}}}\right| \text {, }
$$

or

$$
\tau_{\mathrm{w},-7}=3.2 \times 10^{15}\left(\frac{M_{\mathrm{S}}}{10 M_{\odot}}\right)\left(\frac{\left|\dot{M}_{\mathrm{w}}\right|}{10^{-7} M_{\odot} \mathrm{yr}^{-1}}\right)^{-1} \mathrm{~s},
$$

where we have used the subscript " -7 " to denote the exponent in the wind mass-loss rate.

This characteristic timescale is compared to the dynamical timescales in Figure 1, for a $10 M_{\odot}$ star and for a wind massloss rate of $10^{-7} M_{\odot} \mathrm{yr}^{-1}$, which is consistent with current observations of the star S2 in orbit around Sgr A* (Martins et al. 2008). The effect of wind mass loss becomes negligible with respect to the frame-dragging-induced precession of the orbital planes for orbits within $\sim 30,000$ gravitational radii. On the other hand, they become negligible with respect to the quadrupole-induced precession of the orbital planes for orbits within $\sim 4000$ gravitational radii.

\subsection{Tidal Dissipation of Orbital Energy}

The tidal deformations excited at each periastron passage transfer some of the orbital energy into modes within the volume 
of the star (see Alexander 2006 for a review of stellar processes around Sgr A*). Since the orbital energy loss is proportional to the number of passages (Li \& Loeb 2013), we can use the approach of Press \& Teukolsky (1977) to estimate the rate of dissipation of orbital energy as

$$
\frac{\Delta E}{\Delta t} \simeq\left(\frac{G M_{\mathrm{S}}^{2}}{P R_{\mathrm{S}}}\right)\left(\frac{M_{\mathrm{BH}}}{M_{\mathrm{S}}}\right)^{2} \sum_{l=2,3, \ldots}\left(\frac{R_{\mathrm{S}}}{R_{\mathrm{p}}}\right)^{2 l+2} T_{l}(\eta) .
$$

Here $R_{\mathrm{p}}=a(1-e)$ is the periastron distance, $R_{\mathrm{S}}$ is the radius of the star, and $T_{l}(\eta)$ are appropriate dimensionless functions of the quantity

$$
\eta \equiv\left(\frac{M_{\mathrm{S}}}{M_{\mathrm{S}}+M_{\mathrm{BH}}}\right)^{1 / 2}\left(\frac{R_{\mathrm{p}}}{R_{\mathrm{S}}}\right)^{3 / 2}
$$

that describe the excitation of modes with different spherical harmonic index $l$.

In detail,

$$
T_{l}(\eta)=2 \pi^{2} \sum_{n, m}\left|Q_{n l}\right|^{2}\left|K_{n l m}\right|^{2},
$$

where $n$ is the mode order and $m$ is the other spherical harmonic index. The excited modes have $l>1$ and $-l<m<l$. The coefficient $K_{n l m}$ represents the coupling to the orbit:

$$
K_{n l m}=\frac{W_{l m}}{2 \pi} \int_{-\infty}^{\infty} d t\left[\frac{R_{p}}{r(t)}\right]^{l+1} \exp \left\{i\left[\omega_{\mathrm{n}} t+m \Phi(t)\right]\right\},
$$

where $r(t)$ is the instantaneous distance between the star and Sgr A*, $\omega_{n}$ is the mode frequency, $\Phi(t)$ is the true anomaly, and

$$
\begin{aligned}
W_{l m}= & (-1)^{(l+m) / 2}\left[\frac{4 \pi}{(2 l+1)}(l-m) !(l+m) !\right]^{1 / 2} \\
& \times\left[2^{l} \frac{(l-m)}{2} ! \frac{(l+m)}{2} !\right]^{-1} .
\end{aligned}
$$

The tidal overlap integral $Q_{n l}$ represents the coupling of the tidal potential to a given mode, i.e.,

$$
Q_{n l}=\int_{0}^{1} R^{2} d R \rho(R) l R^{l-1}\left[\xi_{n l}^{\mathcal{R}}+(l+1) \xi_{n l}^{\mathcal{S}}\right]
$$

where $\rho(R)$ is the stellar density profile as a function of radius $R$ and $\xi(R)=\left[\xi_{n l}^{\mathcal{R}}(R) \hat{e}_{R}+\xi_{n l}^{\mathcal{S}}(R) R \nabla\right] Y_{l m}(\theta, \phi)$ is the mode eigenfunction, with $\xi_{n l}^{\mathcal{R}}$ and $\xi_{n l}^{\mathcal{S}}$ being its radial and poloidal components, respectively. We obtain the appropriate stellar density profile from the MESA code (Paxton et al. 2011) and compute the mode eigenfunctions with the ADIPLS code (Christensen-Dalsgaard 2008).

Because the energy gain in each passage depends on $\left(R_{S} / R_{p}\right)^{2 l+2}$ and the values of $Q_{n l}$ and $K_{n l m}$ are similar for modes with different values of $l$, the quadrupole $(l=2)$ modes gain the most energy during the tidal excitation (the $l=0$ and $l=1$ modes are not excited). For this reason, we focus, hereafter, on the $l=2$ modes.
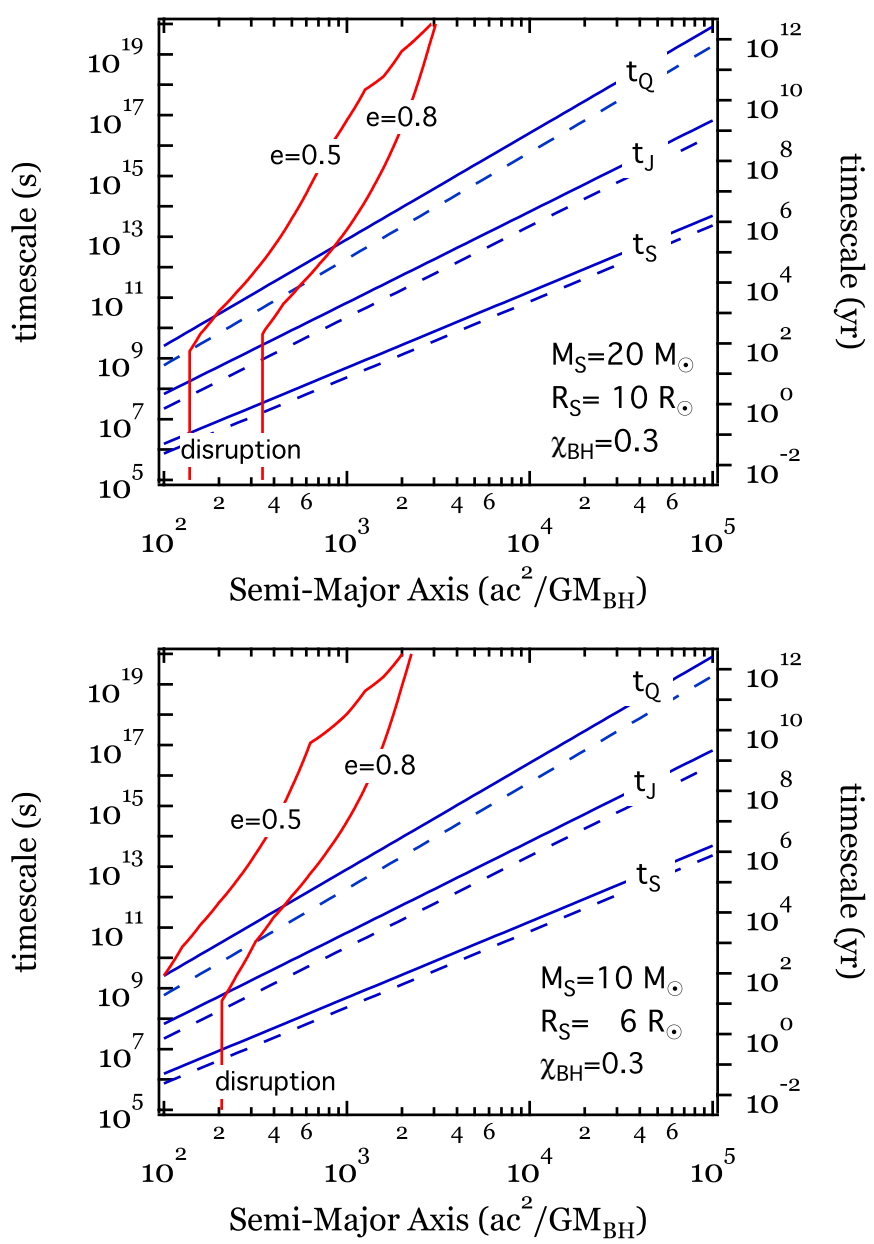

Figure 2. Blue lines show the dynamical timescales, as in Figure 1. The red lines show the characteristic timescale for orbital evolution due to the tidal dissipation of the orbital energy, for two different values of the eccentricity. The vertical segments of the red lines indicate the semimajor axes at which the stars are tidally disrupted at periastron. The two panels correspond to a $20 M_{\odot}$ and a $10 M_{\odot}$ star. In both cases, the black hole spin is taken to be equal to $\chi=0.3$.

(A color version of this figure is available in the online journal.)

The characteristic timescale for orbital evolution due to tidal dissipation is

$$
\begin{aligned}
t_{\mathrm{d}} \equiv & \frac{E}{\Delta E / \Delta t} \\
= & \frac{\pi R_{\mathrm{S}}}{c}\left(\frac{G M_{\mathrm{BH}}}{c^{2} R_{\mathrm{S}}}\right)^{6}\left(\frac{M_{\mathrm{S}}}{M_{\mathrm{BH}}}\right)\left(\frac{a c^{2}}{G M_{\mathrm{BH}}}\right)^{13 / 2}(1-e)^{6} T_{2}^{-1} \\
= & 1.37 \times 10^{-4}\left(\frac{M_{\mathrm{BH}}}{4 \times 10^{6} M_{\odot}}\right)^{5}\left(\frac{R_{\mathrm{S}}}{10 R_{\odot}}\right)^{-5}\left(\frac{M_{\mathrm{S}}}{20 M_{\odot}}\right) \\
& \times\left(\frac{a c^{2}}{G M_{\mathrm{BH}}}\right)^{13 / 2}(1-e)^{6} T_{2}^{-1}(\eta) \mathrm{s},
\end{aligned}
$$

and is shown in Figure 2 for two main-sequence stars with masses $10 M_{\odot}$ and $20 M_{\odot}$.

If the star at periastron reaches inside the tidal radius

$$
R_{\mathrm{t}}=R_{\mathrm{S}}\left(\frac{M_{\mathrm{BH}}}{M_{\mathrm{S}}}\right)^{1 / 3},
$$

it gets disrupted. For simplicity, we ignore here the fact that, if the periastron distance is smaller than four to five times the 


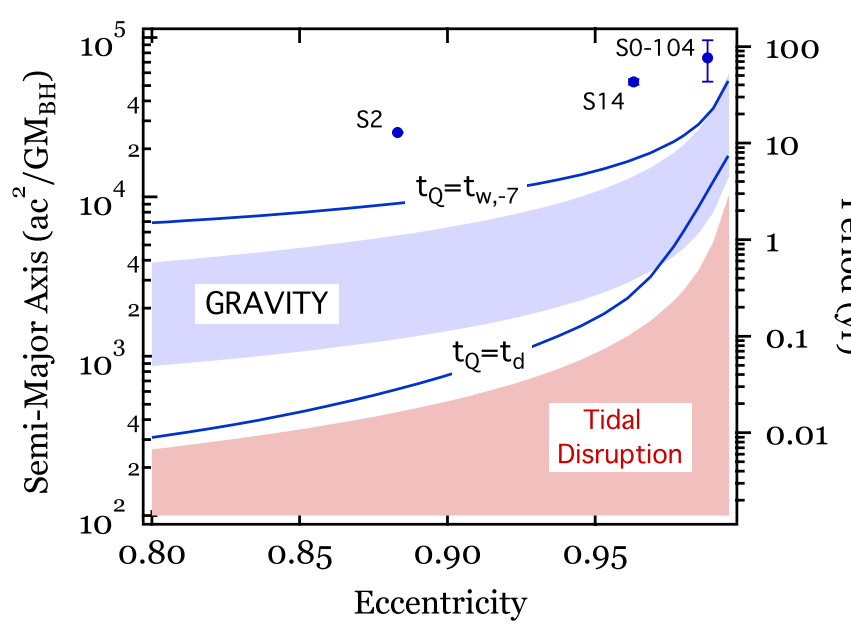

Figure 3. Two blue curves show the loci of orbital parameters for stars around Sgr A* at which the timescale of orbital-plane precession due to the quadrupole moment of the black hole $\left(t_{\mathrm{Q}}\right)$ is equal to the orbital evolution timescale due to stellar winds $\left(t_{\mathrm{w},-7}\right)$ or due to tides $\left(t_{\mathrm{d}}\right)$. In order for stars to follow nearly test-particle trajectories, their orbital parameters have to lie between the two curves. The blue shaded area shows the range of orbital parameters for which frame dragging will be detectable with GRAVITY at a signal-to-noise ratio of 5 , assuming a range of astrometric accuracies between 10 and $200 \mu \mathrm{arcsec}$. The red shaded area shows the range of orbital parameters that leads to the tidal disruption of the star at periapsis. All curves are for a black hole spin of $\chi=0.3$ and a $10 M_{\odot}$ star. The three filled circles show the orbital parameters of the three stars nearest to Sgr A* that are presently known.

(A color version of this figure is available in the online journal.)

tidal radius, the repeated heating of the star at each passage will make it vulnerable to tidal disruption (Li \& Loeb 2013). Requiring $R_{\mathrm{p}} \geqslant R_{\mathrm{t}}$ sets a lower limit on the semimajor axis of the stellar orbit, i.e.,

$$
\begin{aligned}
\left(\frac{a c^{2}}{G M_{\mathrm{BH}}}\right) \geqslant & \frac{68.9}{1-e}\left(\frac{R_{\mathrm{S}}}{10 R_{\odot}}\right) \\
& \times\left(\frac{M_{\mathrm{BH}}}{4 \times 10^{6} M_{\odot}}\right)^{-2 / 3}\left(\frac{M_{\mathrm{S}}}{20 M_{\odot}}\right)^{-1 / 3} .
\end{aligned}
$$

The tidal limit is shown as the vertical portion of the red lines in Figure 2. At orbital separations larger than this limit, the tidal evolution of the stellar orbits is never fast enough to compete with the precession of the orbital planes due to frame dragging. On the other hand, the orbital plane precession due to the quadrupole moment of the black hole for stars with semimajor axes a few times larger than the tidal limit will be masked by the orbital evolution due to tidal effects.

\section{DISCUSSION}

We explored whether deviations of the orbits of star around Sgr A* from test particle trajectories due to stellar winds and tides may compromise the measurements of relativistic effects. Figure 3 summarizes our results for an illustrative case of a $10 M_{\odot}$ star and a black hole spin of $\chi=0.3$. The two blue curves in this figure show the combinations of semimajor axes and orbital eccentricities for which the timescale of orbital plane precession due to the quadrupole moment of the black hole is equal to the orbital evolution timescale due to the wind mass $\operatorname{loss}\left(t_{\mathrm{w}}=t_{\mathrm{Q}}\right)$ and due to tides $\left(t_{\mathrm{d}}=t_{\mathrm{Q}}\right)$. In order for a stellar orbit not to be affected significantly by either of the two effects, its parameters need to be between the two curves.

For comparison, we calculate the signal-to-noise ratio at which the precession of the orbital plane of a star due to frame dragging will be detected, in the near future, using the adaptiveoptics-assisted interferometer GRAVITY. Following Weinberg et al. (2005), we write the signal-to-noise ratio as

$$
S=\frac{8 \pi \chi}{a^{1 / 2}(1+e)^{1 / 2}(1-e)^{3 / 2}}\left(\frac{G M_{\mathrm{BH}}}{D c^{2}}\right)^{3 / 2} \frac{N_{\mathrm{orb}} \cos \psi}{\delta \theta},
$$

where $D$ is the distance to the black hole, $N_{\text {orb }}$ is the number of orbits monitored, $\cos \psi$ is the inclination of the orbit, and $\delta \theta$ is the astrometric accuracy of each measurement. Assuming that we monitor a particular orbit for a time $\Delta T$, we can rewrite this expression as

$$
\begin{aligned}
S= & \frac{9 \times 10^{6} \cos \psi}{(1+e)^{1 / 2}(1-e)^{3 / 2}}\left(\frac{\chi}{0.3}\right)\left(\frac{\Delta T}{10 \mathrm{yr}}\right)\left(\frac{D}{8.4 \mathrm{kpc}}\right)^{-1} \\
& \times\left(\frac{\delta \theta}{10 \mu \operatorname{arcsec}}\right)^{-1}\left(\frac{a c^{2}}{G M_{\mathrm{BH}}}\right)^{-2} .
\end{aligned}
$$

The astrometric accuracy of GRAVITY is expected to be $\sim 200 \mu$ arcsec for a faint star of $m_{\mathrm{K}} \simeq 18.8$ and $\sim 10 \mu$ arcsec for a brighter star of $m_{\mathrm{K}}=16.3$. Requiring a signal-to-noise ratio of 5 for this range of astrometric accuracies and for the typical parameters used in the above equation places an upper limit on the semimajor axes of orbits as a function of their eccentricity. This range of upper limits is shown as the blue-shaded region in Figure 3.

For all but the most eccentric orbits for which GRAVITY will be able to detect orbital-plane precession due to frame dragging, both effects of stellar winds and tides do not preclude by themselves the measurement of the quadrupole moment of the black hole. On the other hand, for highly eccentric orbits $(e>0.96)$, the tidal dissipation of orbital energy for massive stars occurs at similar timescales as the orbital-plane precession due to the quadrupole moment of the black hole. Of course, the range of semimajor axes of orbits for which plane precession will be detected at a particular signal-to-noise ratio depends on the spin of the black hole (see Equation (24)). Given that, in the last equation, we required only a signal-to-noise ratio of 5 and used optimal uncertainties in the positional determination of the stars, it is unlikely that, for black hole spins that are significantly smaller than our fiducial value $(\chi=0.3)$, orbital precession caused by the quadrupole moment of the spacetime will be detectable with GRAVITY.

It is worth emphasizing here that neither stellar winds nor tidal dissipation of orbital energy will lead to precession of the orbital planes of stars, unless a characteristic vector in the system, such as the stellar angular velocity, is misaligned with the angular momentum of the orbit (see, e.g., Eggleton et al. 1998). However, the astrometric observations of stars in orbit around Sgr A* will not be of sufficient quality to distinguish between the change in the position of a star due to precession of its orbital plane and the change in the position due to, e.g., orbital decay. Because of the limited telescope time and the rather significant error bars in each measurement, at most a few data points will be collected before and after the periapsis in an orbit, with error ellipses as large as $200 \mu$ arcsec. If the semimajor axis of the orbit is shrinking because of tidal dissipation but the data are instead fit with an orbit that is only allowed to precess, then the resulting measurement will be highly biased. In this example, the fit procedure would assign the decay of the semimajor axis to a small precession of the orbital plane, such that the projected semimajor axis on the plane of the sky is reduced. For this reason, any orbital evolution caused by non-gravitational effects, 
such as those discussed here, needs to be taken into account as a possible source of systematic uncertainties in measuring the quadrupole moment of the black hole and in testing the no-hair theorem.

We thank S. Gillessen and C. Will for their comments on the manuscript and F. Özel for many constructive discussions and comments. D.P. acknowledges the support of NSF CAREER award AST-0746549. This work also was supported in part by NSF grant AST-0907890 and NASA grants NNX08AL43G and NNA09DB30A (to A.L.).

\section{REFERENCES}

Alexander, T. 2006, JPhCS, 54, 243

Christensen-Dalsgaard, J. 2008, Ap\&SS, 316, 113

Eggleton, P. P., Kiseleva, L. G., \& Hut, P. 1998, ApJ, 499, 853

Eisenhauer, F., Perrin, G., Brandner, W., et al. 2011, Msngr, 143, 16

Fish, V. L., \& Doeleman, S. S. 2010, in IAU Symp. 261, Relativity in Fundamental Astronomy Dynamics, Reference Frames, and Data Analysis, ed. S. Klioner, P. K. Seidelmann, \& M. Soffel (Cambridge: Cambridge Univ. Press), 271

Genzel, R., Eisenhauer, F., \& Gillessen, S. 2010, RvMP, 82, 3121

Ghez, A. M., Morris, M. R., Do, T., et al. 2012, in Proc. 12th Marcel Grossmann Meeting on General Relativity, ed. T. Damour, R. T. Jantzen, \& R. Ruffini (Singapore: World Scientific), 420

Ghez, A. M., Salim, S., Weinberg, N. N., et al. 2008, ApJ, 689, 1044

Gillessen, S., Eisenhauer, F., Trippe, S., et al. 2009, ApJ, 692, 1075

Johannsen, T., \& Psaltis, D. 2010, ApJ, 718, 446

Li, G., \& Loeb, A. 2013, MNRAS, 429, 3040

Liu, K., Wex, N., Kramer, M., Cordes, J. M., \& Lazio, T. J. W. 2012, ApJ, 747, 1

Martins, F., Gillessen, S., Eisenhauer, F., et al. 2008, ApJL, 672, L119

Meyer, L., Ghez, A. M., Schödel, R., et al. 2012, Sci, 338, 84

Merritt, D., Alexander, T., Mikkola, S., \& Will, C. M. 2010, PhRvD, 81, 062002

Paxton, B., Bildsten, L., Dotter, A., et al. 2011, ApJS, 192, 3

Pfahl, E., \& Loeb, A. 2004, ApJ, 615, 253

Press, W. H., \& Teukolsky, S. A. 1977, ApJ, 213, 183

Psaltis, D. 2012, ApJ, 759, 130

Sadeghian, L., \& Will, C. M. 2011, CQGra, 28, 225029

Stone, J. M., Eisner, J. A., Monnier, J. D., et al. 2012, ApJ, 754, 151

Weinberg, N. N., Milosavljević, M., \& Ghez, A. M. 2005, ApJ, 622, 878

Will, C. M. 2008, ApJL, 674, L25 